\title{
Imaging Drug-loaded Block Copolymers at Different Tip Forces
}

\author{
Lenny Dao, * Shrirang Ranade, ${ }^{*}$ Natalya Yerina, ** and Sergei Magonov** \\ * Corporate Research \& Advanced Technology Development, Boston Scientific \\ Corporation, One Boston Scientific Place, Natick, MA 01760 USA \\ ** Veeco Instruments, 112 Robin Hill Road, Santa Barbara, CA 93117 USA
}

We have recently utilized atomic force microscopy (AFM) to examine the morphological distribution of drug particles, Palitaxel (PTx), on the surface of several different drugloaded block copolymer films. AFM study of neat block copolymer films and coatings filled with drug particles or a coating should be conducted at different tip forces in order to correctly analyze surface topography or microstructure and composition [1]. We found out that imaging of the copolymer/drug systems with different forces helps to clarify surface and bulk morphology and to recognize different inclusions and fillers that are present in the film. Fig la shows a spun cast copolymer film of Polystyrenepolyisobutylene-polystyrene (SIBS) imaged using different tip forces. The image using low-force reveals more details of the film surface (true topography), while the lamellar morphology of the block copolymer film is better revealed in the higher-force image (true compositional map) [2-3]. This technique of imaging becomes even more useful once the neat block copolymer film is blended with the drug particles, since the presence of a low molecular weigh additive or traces of monomer material can easily lead to the image features similar to those of drug particles. For instance where we examined the blend of $25 \% \mathrm{PTx} / 75 \% \mathrm{PS}-\mathrm{PBA}-\mathrm{PS}$ spun cast film, as shown in Fig $1 \mathrm{~b}$, the higher force images revealed that most of bright features disappeared or changed their contrast, and only a few particles are still seen in the examined area. The two brightest particles are best seen in the phase image at the $350 \mathrm{~nm}$ scale. This finding indicates that the bright particles observed in the high force images do not belong to the block copolymer matrix, which as we know might exhibit drastic contrast variation during imaging at different forces, and they can be assigned to stiffer particles of the drug.

By using different forces as an AFM imaging technique to study a large number of block copolymer films loaded with drug, we have found that there are different "signatures" attributed to how the drug particles segregate from the copolymers. The differences are related to the size of the particles and their distribution as well as their location on the surface or in a near-surface layer. Figure 4 shows several "signatures" which are recognized from both height and phase images at certain optimized force conditions. The particles, which are fully embedded into the coating, can be identified in phase images as the particle is close enough to the native surface that changes in local mechanical properties occur.

In summary, an application of AFM imaging at different tip forces is a crucial technique in the area of characterizing drug particles in drug loaded copolymer films.

\section{References}


[1]S.N. Magonov, Surface Science (1997) 201.

[2]Y. Wang, R. Song, Y. Li, Surface Science 530 (2003)136.

[3]S.N. Magonov, Encyclopedia of Analytical Chemistry, R.A. Myers (Ed.), John Wiley \& Son Ltd, Chichester, 2000

(a)

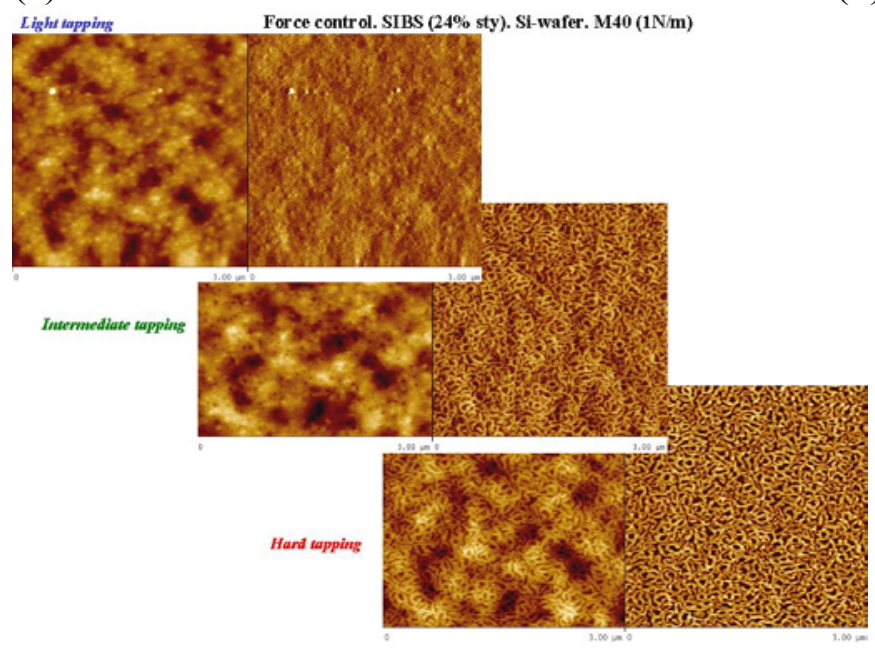

(b)

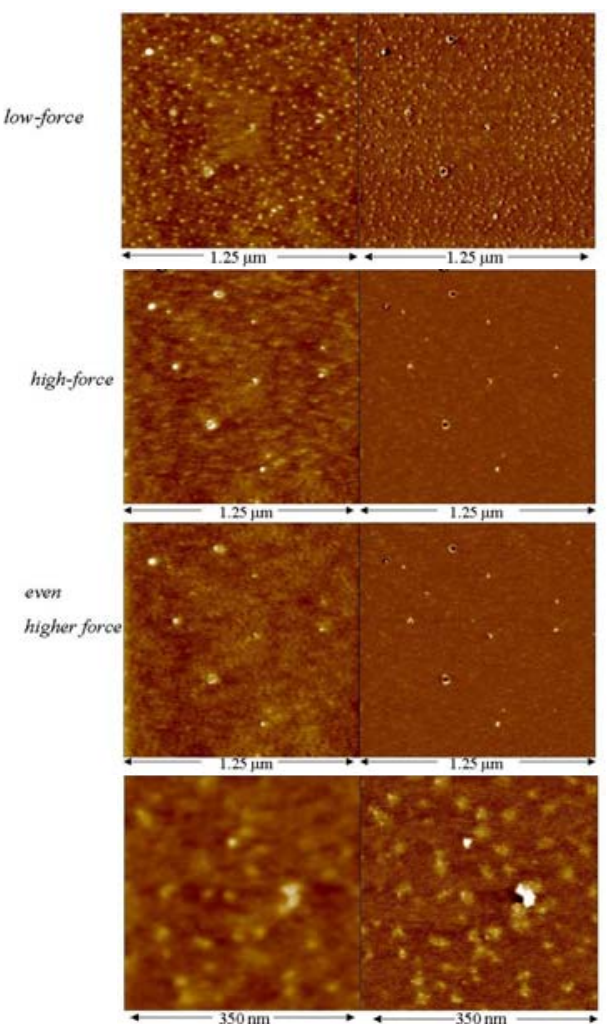

Figure1: Images at different forces of (a) SIBS and (b) 25\%PTx/75\%PS-PBA-PS. Polymer films were solvent cast on Si wafer.
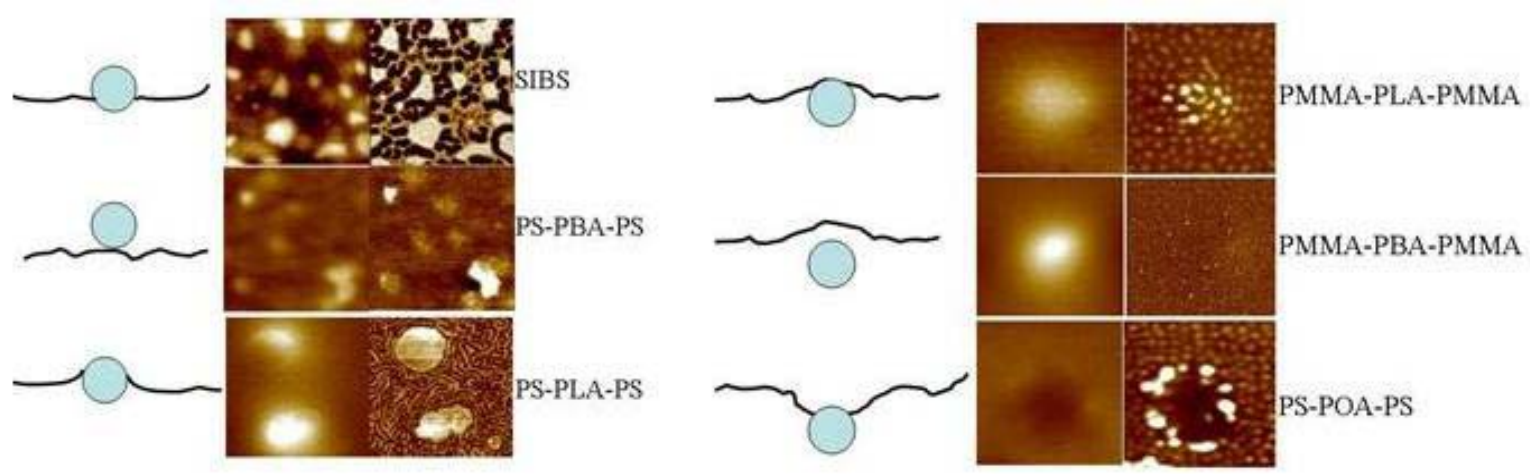

Figure 2: Signatures of drug particles, PTx in copolymer coatings 